\title{
A NOTE ON THE SINGULARITIES OF HARMONIC FUNCTIONS IN THREE VARIABLES
}

\author{
R. P. GILBERT
}

The purpose of this note is to prove the following result:

TheOREM 1. Let $h_{n, m}(X)$ be the homogeneous, harmonic polynomials of degree $n$ defined by the relation

$$
t^{n} \equiv\left[\left(-x_{1}-i x_{2}\right) \frac{\zeta}{2}+x_{3}+\left(x_{1}+i x_{2}\right) \frac{1}{2 \zeta}\right]^{n}=\sum_{m=-n}^{+n} h_{n, m}(X) \zeta^{-m},
$$

and let the analytic function of two complex variables $f(t, \zeta)$, and the three-dimensional harmonic function $H(X)$ be defined by the expansions

$$
\begin{aligned}
f(t, \zeta) & =\sum_{n=0}^{\infty} \sum_{m=-n}^{+n} a_{n m} t^{n} \zeta^{m}, \quad|t|<R, \quad 1-\epsilon<|\zeta|<1+\epsilon, \\
H(X) & =\sum_{n=0}^{\infty} \sum_{m=-n}^{+n} a_{n m} h_{n m}(X), \quad\|X\|<R,
\end{aligned}
$$

which converge in the indicated regions. Furthermore, let the singularities of $f(t, \zeta)$ be given in the form $t=\psi(\zeta)$, where $\psi(\zeta)$ is analytic in $\zeta$. Then $H(X)$ is singular at $X$, providing $X$ does not lie on the $x_{8}$-axis, if and only if $X$ satisfies simultaneously the equations

$$
S(X ; \zeta) \equiv t-\psi(\zeta)=0,
$$

and

$$
\frac{\partial}{\partial \zeta} S(X ; \zeta)=0 .{ }^{1}
$$

Proof. We first note that the harmonic function in three variables $H(X)$, may be generated from the function of two complex variables by the Whittaker-Bergman operator [2],

$$
H(X)=B_{\mathfrak{s}}\left(f, \mathfrak{L}, X^{0}\right) \equiv \frac{1}{2 \pi i} \int_{\mathscr{L}} f(t, \zeta) \frac{d \zeta}{\zeta},
$$

where $t$ is defined as above, $\left\|X-X^{0}\right\|<\epsilon, X \equiv\left(x_{1}, x_{2}, x_{3}\right), X^{0} \equiv\left(x_{1}^{0}, x_{2}^{0}, x_{8}^{0}\right)$, where $\mathcal{L}$ is a regular contour in the $\zeta$-plane, and $\epsilon>0$ is sufficiently

Presented to the Society, April 10, 1961 under the title Singularities of threedimensional harmonic functions. III; received by the editors February 27, 1961.

1 This theorem is the analogue to Nehari's result concerning the singularities of Legendre expansions [1]. 
small. We next consider the following two theorems proved by the author in a previous paper [3].

THEOREM A. If $Z^{3} \equiv E\{S(X ; \zeta)=0\}$, is the singularity manifold of $(1 / \zeta) f(t, \zeta)$, then

$$
H(X)=\frac{1}{2 \pi i} \int_{\mathscr{L}} f(t, \zeta) \frac{d \zeta}{\zeta}
$$

(where $\mathfrak{L}$ is the unit circle) is regular at $X$, providing this point does not satisfy simultaneously the two equations $S(X ; \zeta)=0$, and $(\partial / \partial \zeta) S(X ; \zeta)$ $=0$.

TheOREM B. Let $H(X)=B_{3}(g, \mathcal{L}, \infty)$ be a harmonic function regular at infinity, ${ }^{2}$ and let $V(r, \xi, \rho)$ be the function obtained from $H(X)$ by replacing $x_{1}, x_{2}, x_{3}$ by

$$
\begin{aligned}
& x_{1}=\frac{r}{2}\left(\rho+\rho^{-1}\right)\left(1-\xi^{2}\right)^{1 / 2}, \\
& x_{2}=\frac{r}{2 i}\left(\rho-\rho^{-1}\right)\left(1-\xi^{2}\right)^{1 / 2}, \\
& x_{3}{ }^{-}=r \xi .
\end{aligned}
$$

Furthermore, let $Z^{5} \equiv E\{\Phi(r, \xi, \rho)=0\}$ be the singularity manifold of $V(r, \xi, \rho) \equiv H(X), X \in C^{3}\left\{C^{3}\right.$ is complex three-space $\}$, then the function of two complex variables $g(\tau, \zeta)$, which may be obtained by the reciprocal integral operator

$$
g(\tau, \zeta)=B_{2}\left(V ; \Gamma_{k} ; \tau_{0}, \zeta_{0}\right) \equiv \frac{1}{4 \pi i} \int_{\Gamma_{1}}\left[\int_{\Gamma_{2}} \frac{r(\tau+t)}{(\tau-t)^{2}} V(r, \xi, \rho) \frac{d \rho}{\rho}\right] d \xi
$$

( $\Gamma_{1}$ is the real axis joining -1 to +1 , and $\Gamma_{2}$ is the unit circle), is regular at $(\tau, \zeta)$ providing this point does not lie on the "envelope" of the three parameter family

$$
\tau-t \equiv \tau-r\left[\xi+\frac{i}{2}\left(1-\xi^{2}\right)^{1 / 2}\left(\frac{\zeta}{\rho}+\frac{\rho}{\zeta}\right)\right]=0,{ }^{3}
$$

subject to the auxiliary condition $\Phi(r, \xi, \rho)=0$.

In order to prove Theorem 1 we should like to show that each possible singularity of $H(X)$ (as indicated by Theorem A) corresponds under the reciprocal transform to an actual singularity of $g(\tau, \zeta)$. The possible singularities of $H(X)$ are those

${ }^{2} g(t, \zeta)=\sum_{n-0}^{\infty} \sum_{m-n}^{+n} a_{n m} t^{(n+1)} \zeta^{m}$.

$3 r, \xi$, and $\rho$ are complex spherical coordinates, defined by $r=+\left(x_{1}^{2}+x_{2}^{2}+x_{3}^{2}\right)^{1 / 2}$, $\xi=x_{3} / r$, and $\rho=+\left(\left(x_{1}+i x_{2}\right) /\left(x_{1}-i x_{2}\right)\right)^{1 / 2}$. 


$$
X \in\left[E\{S(X ; \zeta)=0\} \cap E\left\{\frac{\partial S(X ; \zeta)}{\partial \zeta}=0\right\}\right] \equiv E\{F(X)=0\}
$$

The possible singularities of a $g(\tau, \zeta)$ generated by the operator $B_{2}\left(V ; \Gamma_{k} ; \tau_{0}, \zeta_{0}\right)$ (where $V(r, \xi, \rho)$ is a harmonic function with the singularity manifold $E\{\Phi(r, \xi, \rho)=0\} \equiv E\{F(X)=0\})$ are those

$$
\begin{aligned}
(\tau, \zeta) \in[ & E\left\{\tau=-\left(x_{1}-i x_{2}\right) \frac{\zeta}{2}+x_{8}+\left(x_{1}+i x_{2}\right) \frac{1}{2 \zeta}\right\} \\
& \cap E\{F(X)=0\} \\
& \cap E\left\{-\left(1+i \frac{\partial x_{2}}{\partial x_{1}}\right) \frac{\zeta}{2}+\frac{\partial x_{8}}{\partial x_{1}}+\left(1+i \frac{\partial x_{2}}{\partial x_{1}}\right) \frac{1}{2 \zeta}=0\right\} \\
& \cap E\{G(X)=0\}] .4
\end{aligned}
$$

Now, for each fixed $\left.X_{1} \in E\{F(X)=0\}, \quad \tau=-\left(x_{1}-i x_{2}\right)\right\} / 2+x_{3}$ $+\left(x_{1}+i x_{2}\right) 1 / 2 \zeta$ defines $\tau$ as a function of $\zeta, \tau=\phi\left(\zeta \mid X_{1}\right)$; also, since $E\{F(X)=0\} \subset E\{S(X ; \zeta) \equiv t-\psi(\zeta)=0\}$ one has then, in general, for

$$
\begin{aligned}
& X \in[E\{F(X)=0\} \cap E\{G(X)=0\} \\
& \cap E\left\{-\left(1-i \frac{\partial x_{2}}{\partial x_{1}}\right) \frac{\zeta}{2}+\frac{\partial x_{3}}{\partial x_{1}}+\left(1+i \frac{\partial x_{2}}{\partial x_{1}}\right) \frac{1}{2 \zeta}=0\right\},
\end{aligned}
$$

that $\tau=\psi(\zeta) \equiv \phi(\zeta)=t$.

There are some exceptional points (on the $x_{3}$-axis), however, which may not correspond to singularities of $g(t, \zeta)$. This may be seen by considering the method of proof used for Theorems A and B. Both of these results were proven by using a modified form of an idea employed by Hadamard $[1 ; 3 ; 4]$ in the proof of his theorem on the multiplication of singularities. Essentially, the method was to consider the initial domain of definition for a function given in terms of an integral representation, and then to attempt to enlarge this domain by continuously deforming the integration path, subject to the condition that in this process the integration path at no time crosses a singularity of the integrand. One notices from the recip.ocal operator $B_{2}\left(V ; \Gamma_{k} ; \tau^{0}, \zeta^{0}\right)$, that the points $\xi= \pm 1$ then have a special status in that they are fixed endpoints for the path of integration in the $\xi$ plane. Hence, it is quite possible for a branch of $V(r, \xi, \rho) \equiv H(X)$ to have a singularity at $\xi= \pm 1\left(\xi \equiv x_{3} / r\right)$, which does not correspond to a singularity of $g(t, \zeta)$ at $t=\psi(\zeta)$.

4 $G(X)=0$ is an arbitrary relation between $x_{1}, x_{2}$, and $x_{3}$. 


\section{REFERENCES}

1. Z. Nehari, On the singularities of Legendre expansions, J. Rat. Mech. Anal. 5 (1956), 987-992.

2. S. Bergman, Zur Theorie der algebraischen Potentialfunktionen des dreidimensionalen Raumes, Math. Ann. 99 (1928), 629-659.

3. R. Gilbert, Singularities of three-dimensional harmonic functions, Pacific J. Math. 10 (1960), 1243-1255.

4. J. Hadamard, Theorème sur les séries entières, Acta Math. 22 (1898), 55-64.

Michigan State University

\section{AN ALGORITHM OF J. SCHUR AND THE TAYLOR SERIES}

E. H. CONNELL AND P. PORCELLI

In a recent announcement in the Bulletin (cf. [1]), the authors outlined a proof that a differentiable function of a complex variable in a region $R$ has a power series expansion in some neighborhood of each point of $R$ (Theorem 3, [1]). The proof involved no integration, but failed to yield the full radius of convergence and required that it first be shown that all of the derivatives exist. In the last paragraph of [1], it was mentioned that a variation of an algorithm of J. Schur develops constructively the Taylor series for $f$, yields the full radius of convergence, and gives the existence of all the derivatives of $f$ as a corollary. This procedure is developed in this paper.

The letter $R$ will denote a bounded region in the complex plane.

Lemma 1 (cf. $[4$, p. 77$]$ ). If $f: \bar{R}$ into $E_{2}$ is continuous on $\bar{R}$, differentiable on $R$, and nonconstant, then

$$
|f(z)|<\max _{t \in \bar{R}-R}|f(t)| \quad \text { for all } z \in R .
$$

Lemma 2 (Theorem 1, [1]). If $p \in R$ and $f$ is differentiable and bounded on $R-p$, then $f$ may be defined at $p$ so that it will be continuous and differentiable at $p$.

LEMma 3. If the polynomial, $P(z)=a_{0}+a_{1} z+\cdots+a_{n} z^{n}$, satisfies $|P(z)| \leqq 1$ for $|z| \leqq 1$, then $\left|a_{i}\right| \leqq 1, i=0,1, \cdots, n$.

Proof. The conclusion holds for all polynomials of degree zero. Suppose it holds for all polynomials of degree $\leqq k$ and $P(z)=a_{0}$

Received by the editors March 1, 1961. 